\title{
LA PROTECCIÓN DE LOS NIÑOS MIGRANTES EN MÉXICO: UNA FALACIA
}

PROTECTION OF MIGRANT CHILDREN IN MEXICO: A FALLACY

\author{
Gisela María Pérez Fuentes \\ Universidad Juárez Autónoma de Tabasco. México/Mexico \\ giselapef@hotmail.com
}

Recibido/Received: 11/08/2014

Modificado/Modified: 19/08/2014

Aceptado/Accepted: 30/09/2014

\section{RESUMEN}

En el presente artículo se analiza la protección del menor migrante en México cuando se trata de niños que pertenecen a otra ciudadanía y cultura, ello a pesar de la normativa aparentemente protectora en algunos de estos casos, toda vez que en el país hace falta un sistema de protección a este tipo de situación migratoria, que cuente con mecanismos efectivos para enfrentar el problema de fondo y responder eficazmente a la crisis humanitaria que viven los niños provenientes de otros países.

\section{PALABRAS CLAVE}

Interés superior del menor, migración, política pública, derecho mexicano.

\section{SUMARIO}

1. Introducción. 2. Prolegómenos de la protección a los menores en México. 3. Derecho comparado. 4. Marco jurídico del interés superior del menor como derecho fundamental. 5. Ley para la protección de los derechos de niñas, niños y adolescentes. 6. El interés superior del menor en la Suprema Corte de Justicia de la Nación. 7. Niños migrantes. Situación jurídica y problemática en México. 8. Conclusiones. Bibliografía.

\begin{abstract}
In this article the protection of migrant children is discussed in Mexico when it comes to children belonging to other citizenship and culture, in spite of the apparently protective legislation in some of these cases, since the country needs a system protection to this type of immigration status that has effective mechanisms to address the underlying problem and respond effectively to the humanitarian crisis that children from other countries live.
\end{abstract}

\section{KEYWORDS}

Best interests of the child, migration, public policy, Mexican law.

\section{CONTENTS}

1. Introduction. 2. Preliminaries of protection for children in Mexico. 3. Comparative Law. 4. Legal framework of interests of the child as a fundamental right. 5. Act to protect the rights of children and adolescents. 6. The interest of the child in the Supreme Court of Justice. 7. Migrant children. Status and issues in Mexico. 8. Conclusions. References. 


\section{INTRODUCCIÓN}

Desde hace más de una década en el sistema jurídico mexicano se trata de fortalecer al menos jurídicamente, desde los principios de la Constitución mexicana la protección de los derechos humanos, ello a partir de importantes reformas constitucionales en las que se rescata y consolida el principio pro-homine, tal fenómeno jurídico ha tenido gran manifestación en la protección al menor a través del desarrollo del principio del interés superior de éste.

Sin embargo, la realidad social ha superado este principio desarrollado tanto en normativas nacionales y en los Tratados a los que México se ha adherido cuando el tema incorpora la protección de niños migrantes. En el trabajo se parte de la hipótesis que la protección del menor en México ha sido violentada cuando se trata de niños que pertenecen a otra ciudadanía y cultura, ello a pesar de la normativa aparentemente protectora en algunos de estos casos pues realmente en el país no existe un sistema de protección a este tipo de situación migratoria y el gobierno mexicano no cuenta con mecanismos efectivos tanto para enfrentar el problema de fondo, como para responder eficazmente a la crisis humanitaria que viven los niños migrantes pues el asunto no sólo se soluciona con llenar las lagunas de la ley. El objetivo general de este artículo es identificar el interés superior del menor en casos de niños desprotegidos por razón de origen en el derecho mexicano.

\section{PROLEGÓMENOS DE LA PROTECCIÓN A LOS MENORES EN MÉXICO}

En México se ha entendido la necesidad de actualizar el Derecho tanto privado como constitucional y las normas que lo regulan en vías de enriquecer a la comunidad social y conforme a las condiciones históricas que han marcado la conformación de las normas sobre el derecho de familia (Pérez, 2012:34) en este país.

La defensa de los derechos humanos en la Constitución mexicana y sus últimas reformas en este sentido, conjugado con las diferencias en la conformación y defensa del derecho de familia en México, han permitido nuevas pautas interpretativas que marcan cambios sustanciales, plasmando otros principios interpretativos en el texto constitucional y en algunas legislaciones estatales.

La lógica formal como forma de interpretación judicial ha sido superada en los criterios emitidos por la Suprema Corte de Justicia de la Nación mexicana (Galindo, 2011:10).

La Constitución mexicana marcada en un primer momento por normas políticas y sociales, ha dejado de ser fuente exclusiva de derecho público para convertirse en una ley marco. El 10 de junio de 2011 se publicó en el Diario Oficial de la Federación, el decreto que modifica la denominación del Capítulo I del Título Primero para quedar: De los Derechos Humanos y sus garantías y reforma diversos artículos de la Constitución Política de los Estados Unidos Mexicanos, para incorporar el alcance de los derechos humanos. Por eso, en la Constitución Federal prima el principio pro-persona, la protección a la familia y a los débiles sociales en los que asume el primer lugar la infancia, todo ello por supuesto y al menos, en el ámbito legislativo.

Es innegable que formalmente el Código Civil ha perdido su carácter supletorio con exclusividad pero sólo ello para ascender a categoría constitucional, transformándose así los principios tradicionales del derecho en derecho constitucional (Arce, 1986:59). Así que frente a los críticos del Derecho Civil que explican y sostienen la descodificación y por tanto, la desintegración civil (Acosta, 1989:611), por nuestra parte sostenemos el resurgimiento del 
tronco jurídico común de cualquier sistema jurídico, es decir; el Derecho Civil, a partir de su institución fundamental; la persona, expresado a nivel constitucional como jurisprudencial en México a través del principio pro-homine. Por ejemplo, tenemos las siguientes tesis que sostienen lo siguiente:

1) El principio pro-homine, incorporado en múltiples tratados internacionales, es un criterio hermenéutico que coincide con el rasgo fundamental de los derechos humanos, por virtud del cual debe estarse siempre a favor del hombre e implica que debe acudirse a la norma más amplia o a la interpretación extensiva cuando se trata de derechos protegidos $\mathrm{y}$, por el contrario, a la norma o a la interpretación más restringida, cuando se trata de establecer límites a su ejercicio.(T.C.C., SJF, 2004).

2) El principio pro-homine que implica que la interpretación jurídica siempre debe buscar el mayor beneficio para el hombre, es decir, que debe acudirse a la norma más amplia o a la interpretación extensiva cuando se trata de derechos protegidos $\mathrm{y}$, por el contrario, a la norma o a la interpretación más restringida, cuando se trata de establecer límites a su ejercicio, se contempla en los artículos 29 de la Convención Americana sobre Derechos Humanos y 5 del Pacto Internacional de Derechos Civiles y Políticos, publicados en el Diario Oficial de la Federación el siete y el veinte de mayo de mil novecientos ochenta y uno, respectivamente. Ahora bien, como dichos tratados forman parte de la Ley Suprema de la Unión, conforme al artículo 133 constitucional, es claro que el citado principio debe aplicarse en forma obligatoria (T.C.C., SJF, 2005).

En el sistema jurídico mexicano los Convenios son fuente del derecho, en concreto, y en función del artículo 133 de la Constitución política mexicana. Los tratados internacionales, según ha fijado la jurisprudencia en México; están situados en un escalón infraconstitucional pero supralegal; por ello la importancia de los Convenios firmados por México en materia de menores.

Sin embargo, cabe mencionar que de forma reciente, mediante jurisprudencia por contradicción de tesis, el Poder Judicial de la Federación ha establecido que si bien el primer párrafo del artículo 1o. constitucional reconoce un conjunto de derechos humanos cuyas fuentes son la Constitución y los tratados internacionales de los cuales el Estado Mexicano sea parte. De la interpretación literal, sistemática y originalista del contenido de las reformas constitucionales de seis y diez de junio de dos mil once, se desprende que las normas de derechos humanos, independientemente de su fuente, no se relacionan en términos jerárquicos, entendiendo que, derivado de la parte final del primer párrafo del citado artículo 1o., cuando en la Constitución haya una restricción expresa al ejercicio de los derechos humanos, se deberá estar a lo que indica la norma constitucional, ya que el principio que le brinda supremacía comporta el encumbramiento de la Constitución como norma fundamental del orden jurídico mexicano, lo que a su vez implica que el resto de las normas jurídicas deben ser acordes con la misma, tanto en un sentido formal como material, circunstancia que no ha cambiado; lo que sí ha evolucionado a raíz de las reformas constitucionales en comento es la configuración del conjunto de normas jurídicas respecto de las cuales puede predicarse dicha supremacía en el orden jurídico mexicano. Esta transformación se explica por la ampliación del catálogo de derechos humanos previsto dentro de la Constitución Política de los Estados Unidos Mexicanos, el cual evidentemente puede calificarse como parte del conjunto normativo que goza de esta supremacía constitucional. En este sentido, los derechos humanos, en su conjunto, constituyen el parámetro de control de regularidad constitucional, conforme al cual debe analizarse la validez de las normas y actos que forman parte del orden jurídico mexicano (Pleno, SJF, 2014). 
México ha firmado importantes instrumentos internacionales de protección a los menores, destacando a efectos de este trabajo, los siguientes:

1) Convención sobre los Derechos del Niño. Adoptada y abierta a la firma y ratificación por la Asamblea General en su resolución 44/25, de 20 de noviembre de 1989, aprobada por el Senado el 19 de junio de 1990, según publicación en el Diario Oficial de la Federación el 31 de julio de 1990. El decreto de promulgación se publicó en el citado diario el 25 de enero de 1991. En la Convención se recuerda que en la Declaración Universal de Derechos Humanos, las Naciones Unidas proclamaron que la infancia tiene derecho a cuidados y asistencia especiales.

2) La Convención Americana de 1969 (Pacto de San José). La Convención destaca por una dualidad en el reconocimiento de los derechos humanos, o como señalan algunos autores, derechos para todos y derechos específicos, en los que destacan la de niños, adolescentes o menores (González et al. 2011:71).

3) Corte Interamericana de Derechos Humanos. La Corte Interamericana es una institución judicial autónoma de la OEA, su objetivo es la aplicación e interpretación de la Convención Americana sobre Derechos Humanos y de otros tratados concernientes al mismo asunto y fue establecida en 1979, está formada por juristas de la más alta autoridad moral y reconocida competencia en materia de derechos humanos elegidos a título personal.

Existen otros instrumentos internacionales que integran el sistema interamericano de derechos humanos de niñas, niños y adolescentes, destacan entre ellos:

1) Convención Interamericana sobre Conflictos de Leyes en Materia de Adopción de Menores. OEA, 24 de mayo de 1984. Fue ratificada por México el 12 de junio de 1987; para entrar en vigor el 26 de mayo de 1988.

2) Convención Interamericana sobre Restitución Internacional de Menores. Firmada por la OEA, el 15 de julio de 1989. Ratificada por México el 5 de octubre de 1994; entrada en vigor en México: 4 de noviembre de 1994.

3) Convención Interamericana Sobre Obligaciones Alimentarias. OEA, 24 de mayo de 1984. Entró en vigor en México el 26 de mayo de 1988, que emitió la declaración interpretativa siguiente: El Gobierno de México, declara de conformidad con el artículo 3 de la Convención que reconoce como acreedores alimentarios además de los señalados, a los concubinos, a los parientes colaterales dentro del cuarto grado menores o incapaces y al adoptado en relación con el adoptante. La obligación de dar alimentos es recíproca. El que los da tiene a su vez el derecho de pedirlos.

\section{DERECHO COMPARADO}

En países europeos del sistema romano germano, como España, se reconoce el derecho a la protección de la juventud y de la infancia por ser colectivo vulnerables. Mediante Instrucción 2/2006 del Fiscal General del Estado, se determinó que para armonizar el derecho a informar y los derechos del menor habrá de partirse que estará justificada la difusión de información veraz y de interés público aunque afecte a un menor siempre que no sea contrario a sus intereses o se garantice su anonimato.

En la investigación de Derecho Comparado sobre el tema de protección de los menores a partir del interés superior, detectamos como se explica a continuación un vacío en la protección de la niñez en caso del debido proceso y de la actuación de los medios, resultó de suma importancia, la solución que otorga el Manual sobre la justicia en asuntos concernientes a los niños víctimas y testigos de delitos para uso de profesionales y encargados de la 
formulación de políticas (ONU, 2010) en cuanto a la manera de aplicar las directrices sobre la justicia, considerando como actores con obligaciones a jueces, servidores públicos, abogados, legisladores y principalmente medios de comunicación.

Por ejemplo, en caso de los jueces, se establece la obligación de respetar la confidencialidad de la información sobre niños víctimas y testigos de delitos; cuando sea necesario para salvaguardar la intimidad de los niños víctimas o testigos, ordenar la exclusión total del público y celebrar vistas a puerta cerrada.

En el caso de Servidores Públicos, se impone la obligación de respetar la confidencialidad de la información sobre niños víctimas y testigos de delitos, en particular absteniéndose de revelar esta información a ninguna persona sin la previa autorización.

Tratándose de Abogados, deberán respetar la confidencialidad de la información sobre niños víctimas y testigos de delitos, en particular absteniéndose de revelar esta información a ninguna persona sin la previa autorización.

Por lo que se refiere a medios de comunicación, deberán adoptar y respetar medidas de autorregulación para proteger la intimidad y los datos personales de una víctima y testigos de delitos; fomentar la aplicación de códigos de conducta.

\section{MARCO JURÍDICO DEL INTERÉS SUPERIOR DEL MENOR COMO DERECHO FUNDAMENTAL}

En un cambio paradigmático, la Constitución Política de los Estados Unidos Mexicanos reconoce y protege los derechos de niñas, niños y adolescentes, destacando entre las modificaciones, las siguientes: se incorpora el concepto de "niño" en la pretensión de sustituir paulatinamente el término "menor"; se establece la obligación del Estado de proveer lo necesario para el respeto a la dignidad de la niñez y la efectividad en el ejercicio de sus derechos, y se considera el deber de los padres, tutores y custodios de preservar tales derechos.

Para 2001 se realizan importantes reformas a la Constitución, en el artículo 1o. se establece la prohibición de toda discriminación. Como ya se ha mencionado, la reforma constitucional del 10 de junio de 2011 incorporó a la carta magna "los derechos humanos", en sustitución de "las garantías individuales", con ello se incorporan los derechos contenidos en los tratados internacionales de derechos humanos de los que México es Estado parte, lo cual se traduce en que el conjunto de derechos y obligaciones reconocidos a nivel internacional formen parte del orden jurídico nacional. Esto implica la aplicación de los principios de pro persona o "interpretación conforme".

Atendiendo al contenido del artículo 1o. de la Constitución se debe entender que la Convención sobre Derechos del Niño es derecho interno, por lo que los derechos de los niños, niñas y adolescentes se ampliaron a partir del 10 de junio de 2011.

Por último, posterior a la reforma constitucional en materia de derechos humanos, el $12 \mathrm{de}$ octubre de 2011 se reforma el artículo 4o. constitucional en el que se incorpora el principio del interés superior de la niñez, así como su incorporación en las políticas públicas del gobierno de México y que tanto el Congreso Federal como los congresos locales pueden legislar en la materia.

El término "interés superior del menor" sigue siendo no obstante, una cláusula abierta y que corresponde a los tribunales definir ponderadamente y no arbitrariamente, el contenido 
de tal principio, sobre este particular se ha pronunciado también el Poder Judicial de la Federación en México, en los siguientes términos:

La expresión interés superior del niño implica que el desarrollo de éste y el ejercicio pleno de sus derechos deben ser considerados como criterios rectores para la elaboración de normas y la aplicación de éstas en todos los órdenes relativos a la vida del niño ( $1^{\mathrm{a}}$ sala, SJF, 2007).

El sistema jurídico mexicano establece diversas prerrogativas de orden personal y social en favor de los menores, lo que se refleja tanto a nivel constitucional como en los tratados internacionales y en las leyes federales y locales (T.C.C., SJF, 2011).

El principio del interés superior del menor se ha incorporado a las leyes civiles en los diferentes Estados del país. Se ha considerado en primer lugar la ley federal que será analizada a continuación.

\section{LEY PARA LA PROTECCIÓN DE LOS DERECHOS DE NIÑAS, NIÑOS Y ADOLESCENTES}

La ley fue publicada en el Diario Oficial de la Federación el 29 de mayo de 2000, cuya última reforma es del día 19 de agosto de 2010; dicha ley se fundamenta en un principio constitucional que supera el tradicional derecho civil, pues en el párrafo sexto del artículo 4o. de la Constitución Política de los Estados Unidos Mexicanos, aparecen disposiciones de orden público, interés social y de observancia general en toda la República Mexicana y tiene por objeto garantizar a niñas, niños y adolescentes la tutela y el respeto de los derechos fundamentales reconocidos en la Constitución.

La Ley define en lenguaje incluyente el concepto de niños y niñas. De acuerdo al artículo 2, son niñas y niños las personas de hasta 12 años incompletos, y adolescentes los que tienen entre 12 años cumplidos y 18 años incumplidos.

La protección de los derechos de niñas, niños y adolescentes, tiene como objetivo asegurarles un desarrollo pleno e integral, lo que implica la oportunidad de formarse física, mental, emocional, social y moralmente en condiciones de igualdad.

El artículo 3 de la ley que se analiza, establece que son principios rectores de la protección de los derechos de niñas, niños y adolescentes:

A. El del interés superior de la infancia.

B. El de la no-discriminación por ninguna razón, ni circunstancia.

C. El de igualdad sin distinción de raza, edad, sexo, religión, idioma o lengua, opinión política o de cualquier otra índole, origen étnico, nacional o social, posición económica, discapacidad, circunstancias de nacimiento o cualquiera otra condición suya o de sus ascendientes, tutores o representantes legales.

D. El de vivir en familia, como espacio primordial de desarrollo.

E. El de tener una vida libre de violencia.

F. El de corresponsabilidad de los miembros de la familia, Estado y sociedad.

G. El de la tutela plena e igualitaria de los derechos humanos y de las garantías constitucionales.

La Ley también define el contenido del principio del interés superior de la infancia, considerando que las normas aplicables a niñas, niños y adolescentes, se entenderán dirigidas a procurarles, primordialmente, los cuidados y la asistencia que requieren para lograr un crecimiento y un desarrollo plenos dentro de un ambiente de bienestar familiar y social. Atendiendo a este principio, el ejercicio de los derechos de los adultos no podrá, en ningún 
momento, ni en ninguna circunstancia, condicionar el ejercicio de los derechos de niñas, niños y adolescentes.

Los Principios Generales precisamente se aplicarán a falta de disposición expresa en la Constitución, en esta ley o en los tratados internacionales en los términos del artículo 133 de la Constitución.

\section{EL INTERÉS SUPERIOR DEL MENOR EN LA SUPREMA CORTE DE JUSTICIA DE LA NACIÓN}

De conformidad con la Constitución Política de los Estados Unidos Mexicanos, diversas normas internacionales y otras más de derecho interno, que consagran el principio del interés superior de la niñez; el niño tiene derecho a preservar su identidad, el nombre y las relaciones familiares, así como prestar asistencia y cuidado cuando se le prive de alguno de los elementos de identidad, para restablecérselo de inmediato, pues el régimen de derechos contiene un verdadero sistema proteccionista, al respecto se ha pronunciado en varios asuntos, en los que se destaca:

El derecho de identidad del menor frente al acto personalísimo de reconocimiento de filiación.

En este orden de ideas, el reconocimiento voluntario ante el funcionario del Registro Civil, de un hijo como propio, es un acto jurídico personalísimo, merced al cual, el compareciente y el reconocido adquieren todos los derechos y obligaciones que atribuye la filiación, y dado que la ley no exige al presunto padre, prueba de la paternidad para llevar a cabo el acto del reconocimiento, es factible hacerlo, tanto en el caso de que no exista vínculo consanguíneo alguno, como cuando exista duda, o inclusive cuando aquel que se presenta a reconocer, goce de elementos que le den certeza de que el reconocido es su verdadero descendiente. Así, la acción de revocación de la filiación reconocida en el acta de nacimiento, respecto de un menor habido fuera de matrimonio, es improcedente, ya que cuando la pretensión sea impugnar tal reconocimiento, es preciso demandar la nulidad de dicho acto jurídico, pues no basta demostrar la falta de vínculo biológico.

En estos casos es indispensable acreditar la nulidad del reconocimiento; esto es, la falta de una verdadera declaración de paternidad emitida por persona con la capacidad que la ley exige o la circunstancia de que al efectuarse hubiera mediado error, engaño, violencia física, intimidación; actualizando un vicio del consentimiento en el reconocimiento del menor ante la oficina del Registro Civil.

Lo anterior señalado no contraviene el principio de irrevocabilidad del reconocimiento de un hijo pues, éste al igual que cualquier acto jurídico es susceptible de su nulidad, pero no debe confundirse la anulación decretada vía sentencia judicial, con la revocación del reconocimiento a manera de retractación (T.C.C., SJF, 2010).

\section{NiÑOS MIGRANTES. SITUACIÓN JURÍDICA Y PROBLEMÁtICA EN MÉXICO}

En el año 2003, México ratificó el Protocolo para Prevenir, Reprimir y Sancionar la Trata de Personas, Especialmente Mujeres y Niños, el cual sentó las bases para la publicación de la Ley para Prevenir y Sancionar la Trata de Personas, en noviembre de 2007, y su Reglamento, 
en febrero de 2009. Los 31 estados de la República Mexicana y el Distrito Federal, han tipificado el delito de la trata de personas, estableciendo reformas en sus códigos penales o publicando su propia legislación contra la trata de personas.

Por "trata de personas" se entenderá la captación, el transporte, el traslado, la acogida o la recepción de personas, recurriendo a la amenaza o uso de la fuerza u otras formas de coacción, al rapto, al fraude, al engaño, al abuso de poder o de una situación de vulnerabilidad o a la concesión o recepción de pagos o beneficios para obtener el consentimiento de una persona que tenga autoridad sobre otra, con fines de explotación. Esta explotación incluirá, como mínimo, la explotación de la prostitución ajena u otras formas de explotación sexual, los trabajos o servicios forzados, la esclavitud o las prácticas análogas a la esclavitud, la servidumbre o la extracción de órganos (CIM et al. 2006).

Se incorpora en la normativa mexicana también la trata en tentativa, es decir, se refiere a la falta de consumación del delito; puede incluir el traslado de las personas, o ambas etapas del delito de trata, sin llevarse a cabo la explotación. El término "tentativa" se aplica de forma supletoria según lo expuesto en el Código Penal Federal, artículo 12, donde se refiere que existe tentativa punible, cuando la resolución de cometer un delito se exterioriza realizando en parte o totalmente los actos ejecutivos que deberían producir el resultado, $\mathrm{u}$ omitiendo los que deberían evitarlo, si aquel no se consuma por causas ajenas a la voluntad del agente.

Al año 2010, 12.3 millones de personas serían víctimas de trata en todo el mundo; más de la mitad son mujeres y niñas (G-TiP, 2010). Pero en el movimiento de personas indocumentadas, entre ellas los menores, destaca también la migración. La migración es un hecho social que se refiere a los movimientos de población. En el mundo, según datos de la Organización Internacional de Migración, se calcula que actualmente hay 214 millones de migrantes internacionales (Le Goff et al. 2011:22).

La migración se convierte en una herramienta para las personas que anhelan encontrar mejores condiciones de vida fuera de su lugar de origen o de residencia, sin embargo, en México durante el año 2014 se acrecentó un fenómeno socialmente trágico en el cual tal decisión de traslado la ejecutan personas menores de 18 años, esto es jurídicamente niños. Sobre este fenómeno existe información que de enero a mayo del 2014, ingresaron a México y fueron detenidos 8.007 menores migrantes, según cifras de la Secretaría de Gobernación. De ellos, 5.175 tenían de 12 a 17 años, y el resto, 2.832, de cero a 11 .

El dato representa un promedio de 53 menores al día y un incremento de $129 \%$ respecto del mismo periodo del año anterior, cuando se registró el ingreso y detención de 3.497 menores. El gobierno de México reconoce que en su frontera sur, compartida con Guatemala y Belice, existen numerosos cruces irregulares que son aprovechados por los migrantes -De los 8.007 menores que fueron detenidos en México de enero a mayo pasado, 3.777 lo hicieron acompañados de un adulto y los 4.230 restantes lo hicieron solos-y es que en México, la especificidad del contexto migratorio potencia el vínculo entre la migración y la trata, y veremos que muchas víctimas de la trata previamente han pasado por las redes de tráfico ilícito de migrantes (o redes de "polleros" o "coyotes"), que las ayudaron a cruzar de manera irregular y clandestina las fronteras.

Existen conceptualmente diferencias entre la migración y la trata, la definición de tráfíco de migrantes que establece el artículo $3^{\circ}$ inciso A del Protocolo contra el Tráfico Ilícito de Migrantes por Tierra, Mar y Aire, en donde se entiende como "la facilitación de la entrada ilegal de una persona a un Estado Parte del cual dicha persona no sea nacional o residente permanente con el fin de obtener, directa o indirectamente un beneficio financiero u otro beneficio de orden material. 
En términos relativos a la trata de personas, el principio del interés superior del niño debe aplicarse de manera sistemática en cualquier medida que se tome respecto a los niños, niñas y adolescentes víctimas de este delito. Para ello, se puede partir de las Directrices del Alto Comisionado de las Naciones Unidas para los Refugiados, que abordan el tema de la determinación del interés superior del menor (2008).

Según las Directrices, el principio del interés superior del menor se aplica a dos tipos de acciones: las acciones que afectan a todos los menores de edad o a grupos de personas menores y las acciones que afectan a un(a) niño(a) de forma individual. Ante las acciones que afectan a todos los niños, niñas y adolescentes o a grupos de ellos, el principio se implementa por medio de medidas más bien generales, que tienen que ver con decisiones políticas, como, por ejemplo, la creación e implementación de proyectos, las políticas y programas sobre esta población, la asignación de recursos, entre otras cuestiones. En cuanto a las acciones que afectan a un(a) niño(a) de forma individual, el principio se concreta en medidas que ayuden a identificar el mejor interés del niño.

La situación de este segundo grupo genera especial preocupación entre gobiernos y organizaciones de derechos humanos, por los peligros que estos menores corren en su tránsito, ya sea con funcionarios, delincuentes u otros adultos.

"El incremento de niñas y niños que viajan, solos o con sus familias, ya alcanzó carácter de crisis humanitaria por la cantidad de víctimas, las precarias condiciones de viaje, los crecientes riesgos asociados al crimen organizado transnacional, la corrupción policiaca y migratoria, la impunidad ante las agresiones y especialmente por las omisiones de los gobiernos", señaló recientemente la Red por los Derechos de la Infancia en México (REDIM).

Las políticas migratorias en México deben considerar cuando una persona o institución tiene que realizar acciones que repercutan de manera particular en la vida de una persona menor de edad, debe elaborar una determinación del interés superior del menor. Ésta se hace a través de un proceso diseñado para tomar decisiones relevantes respecto de un menor y debe incluir garantías procesales, asegurar la participación del niño, y tomar en cuenta sus opiniones.

Finalmente, debe involucrar a personas expertas, con facultades para decidir y valorar la mejor opción respecto de la situación de las personas menores de edad. En el marco de los derechos de la infancia deben buscar que las personas menores de 18 años puedan estar protegidas en el trayecto, y cuando llegan a México o a Estados Unidos, ofrecerles las condiciones necesarias para permanecer en el país el tiempo necesario en tanto se garantiza un retorno seguro a sus familias y comunidades", pero el problema de los menores migrantes "no acompañados" se ha convertido en una crisis humanitaria en el cual se aplica una política de criminalización no sólo por parte de Estados Unidos sino también por México.

Durante los primeros meses del 2014, se ha extendido un rumor en los países de América Central que en cuanto lleguen a territorio estadounidense los niños migrantes van a gozar de una protección automática, que se van a reunir con sus padres o familiares, a lo cual sí tienen derecho, sin embargo, eso no les da automáticamente el derecho a permanecer en el territorio de ese país.

El 80\% de los menores migrantes detenidos, aproximadamente 6.462 fue ubicado en sólo cinco estados de México. La entidad donde se detuvo a la mayor cantidad fue Chiapas con 2.922, a la que le siguieron Veracruz con 1.318; Tabasco con 1.065; Oaxaca con 629, y Tamaulipas con 528. 
El gobierno de Estados Unidos informó que en los últimos ocho meses detuvo en la frontera a más de 47 mil niños migrantes "no acompañados", el doble de los reportados ocho meses antes. Mientras que el Instituto Nacional de Migración reportó que en los primeros cinco meses del año las autoridades estadounidenses deportaron a México seis mil 330 niños, principalmente centroamericanos (Senado de la República, 2014).

De los menores migrantes detenidos en los primeros meses del año, el 98\% provenía de Centroamérica: 7.872. El país con el número más alto fue Honduras con 4.241, seguido de Guatemala con 2.123, y de El Salvador con 1.450. Muy por debajo se ubicaron Nicaragua y Belice.

Chiapas comparte con Guatemala casi 1.000 kilómetros de línea fronteriza. Mientras tanto, Veracruz, Tabasco y Oaxaca son entidades del sur mexicano por las cuales los migrantes deben cruzar en su camino al norte. Tamaulipas, por último, es un estado del norte fronterizo con el sur de Texas, en Estados Unidos. De enero a mayo pasado, según Gobernación, fueron devueltos a sus países 6.227 menores migrantes.

Actualmente, cuando un menor es detenido, las autoridades lo trasladan a una de las estaciones a cargo del Instituto Nacional de Migración (INM), que a su vez depende de la Secretaría de Gobernación, donde permanece mientras se define su situación.

Sin embargo, algunos legisladores consideran que los menores migrantes no deben estar en esos espacios porque requieren condiciones diferentes que los migrantes adultos.

En abril, un grupo de cuatro senadoras de varios partidos presentó una iniciativa de reforma a la Ley de Migración, para que los menores no sean enviados a estaciones migratorias, sino a centros de atención a cargo de los sistemas para el Desarrollo Integral de la Familia (DIF), tanto federal como locales.

En 2011 se registraron 4.160 detenciones de niños, niñas y adolescentes migrantes, en 2012 se contabilizaron 6.107, mientras que en 2013 la cifra llegó a 9.893 casos. Esto representa un aumento en las deportaciones entre 2011 y 2013 del 137\%. Por otro lado, hasta junio de este año, el total de menores detenidos registrado por el INM fue de 10.515 niños, esto es, $6 \%$ más en relación al total del año anterior.

\section{CONCLUSIONES}

La protección jurídica del menor en estas diversas normativas ha permitido reconocer como principio el interés superior del menor, lo que implicó nuevas políticas que se concretan en reformas federales acordes con los principios del derecho internacional contemporáneo y que superan el tradicional derecho civil, donde la familia era una célula básica de la sociedad con carácter impenetrable. Los cambios se observan en primer término, en que se busque el beneficio directo del niño o niña integrante de esa familia, conformando el contenido de este principio constitucional que es también propio del Derecho de Familia.

El interés superior del menor ha entrado entonces a formar parte de lo que la Suprema Corte de Justicia de la Nación mexicana ha denominado, "núcleo duro de derechos", identificándose éstos como aquellos que no admiten restricción alguna y, por tanto alcanzan al legislador, cuando en la normativa jurídica se reconocen expresamente el cúmulo de derechos y se dispone el mandato de efectivizarlos, y actualizado el supuesto jurídico para alcanzar la función de aquel principio, surge una serie de deberes que las autoridades estatales tienen que atender, entre los cuales se encuentra analizar, caso por caso, ante situaciones específicas. 
El interés superior del menor como principio garantista, también implica la obligación de priorizar las políticas públicas destinadas a garantizar el "núcleo duro" de los derechos, pero no es todo, se hace imprescindible acudir al método argumentativo de la ponderación, consistente en analizar caso por caso ante situaciones conflictivas donde existan otros intereses de terceros, de forma tal que, el alcance del interés superior del menor deberá fijarse según las circunstancias particulares del caso y no podrá implicar la exclusión de los derechos de terceros.

\section{BIBLIOGRAFÍA}

ACOSTA ROMERO, M. (1989). "El fenómeno de la descodificación en el Derecho Civil" Revista de Derecho Privado. España.

ARCE Y FLORES-VALDÉS, J. (1986). El Derecho Civil-Constitucional. Madrid: Civitas. CASTÁN TOBEÑAS, J. (1954). La ordenación sistemática del Derecho Civil, Madrid: Reus.

CIM, OEA, OIM, INMUJERES, INM, USAID (2006). Trata de Personas. Aspectos Básicos. México: Coeditado por Comisión Interamericana de Mujeres de la Organización de Estados Americanos, Organización Internacional para las Migraciones, Instituto Nacional de Migración, Instituto Nacional de las Mujeres.

GALINDO SIFUENTES, E. (2011). Argumentación Jurídica. México: Porrúa.

GARCÍA MÁYNEZ, E. (2001). Introducción a la lógica jurídica. México: Colofón.

GONZÁLEZ MARTIN, N. y RODRÍGUEZ JIMÉNEZ, S. (2011). El interés superior del menor en el marco de la adopción y el Tráfico Internacional, Contexto Mexicano. México: Instituto de Investigaciones Jurídicas - Universidad Nacional Autónoma de México.

LAUCCI, C. (2010). Manual sobre la justicia en asuntos concernientes a los niños víctimas y testigos de delitos para uso de profesionales y encargados de la formulación de políticas. Nueva York, USA.: UNODC - UNICEF, Oficina de las Naciones Unidas contra la Droga y el Delito - Fondo de las Naciones Unidas para la Infancia, Serie Manuales de Justicia Penal.

LE GOFF, H. et al. (2011). La trata de personas en México: diagnóstico sobre la asistencia a víctimas. México: Organización Internacional para las Migraciones, Misión.

OFICINA DE G-TiP, (2010). Trafficking in Persons Report 2010. Washington, Estados Unidos: Departamento de Estado de Estados Unidos, disponible en http://www.state.gov/j/tip/rls/tiprpt/2010/ 142760.htm

PÉREZ FUENTES, G. et al. (2012). El Derecho en México. Madrid: Ubijus-Zavalia-Temis

ROGEL VIDE, C. (2010). Derecho Civil-método y concepto-. Madrid: Ubijus-Zavalia-Temis. 


\section{Breve currículo:}

\section{Gisela María Pérez Fuentes}

Doctora en derecho, profesora investigadora de tiempo completo en la Universidad Juárez Autónoma de Tabasco. Líder del Cuerpo Académico "Estudios de Derecho Civil". Pertenece al Sistema Nacional de Investigadores Nivel II de CONACYT. Sus líneas de investigación son: la persona, daño moral, derechos de la personalidad, especialmente derecho al honor, intimidad e imagen. Desde el 2012 es responsable del Observatorio de la Información en el Sureste. Ha publicado y coordinado más de 20 libros y 50 artículos científicos en revistas nacionales e internacionales, como España, México, Cuba, Argentina, Bolivia, Chile, Guatemala y Venezuela. 